\title{
Meta
}

Journal des traducteurs

Translators' Journal

\section{Idiomaticité et gestion de données terminologiques : une approche notionnelle}

\section{Marc Van Campenhoudt}

Volume 39, numéro 1, mars 1994

La traduction et l'interprétation dans la Belgique multilingue

URI : https://id.erudit.org/iderudit/002191ar

DOI : https://doi.org/10.7202/002191ar

Aller au sommaire du numéro

\section{Éditeur(s)}

Les Presses de l'Université de Montréal

ISSN

0026-0452 (imprimé)

1492-1421 (numérique)

Découvrir la revue

Citer cet article

Van Campenhoudt, M. (1994). Idiomaticité et gestion de données terminologiques : une approche notionnelle. Meta, 39(1), 97-106. https://doi.org/10.7202/002191ar
Résumé de l'article

En terminographie multilingue, la perspective notionnelle implique des choix si l'on entend décrire l'idiomaticité. L'auteur dresse une typologie des problèmes de traduction posés par les expressions idiomatiques dans le domaine de la navigation et propose de décrire celles-ci comme des notions à par entière. Dans une base de données relationnelles, la présence d'un lien dit idiomatique devrait permettre de lier chaque expression aux termes qu'elle utilise, de manière à faciliter la recherche d'une traduction. 


\title{
IDIOMATICITÉ ET GESTION DE DONNÉES TERMINOLOGIQUES: UNE APPROCHE NOTIONNELLE
}

\author{
MARC VAN CAMPENHOUDT \\ Institut supérieur de traducteurs et interprètes, Bruxelles, Belgique
}

\begin{abstract}
Résumé
En terminographie multilingue, la perspective notionnelle implique des choix si l'on entend décrire l'idiomaticité. L'auteur dresse une typologie des problèmes de traduction posés par les expressions idiomatiques dans le domaine de la navigation et propose de décrire celles-ci comme des notions à part entière. Dans une base de données relationnelles, la présence $d$ ' un lien dit idiomatique devrait permettre de lier chaque expression aux termes qu' elle utilise, de manière à faciliter la recherche d'une traduction.
\end{abstract}

\section{INTRODUCTION}

Depuis quelques années, les recherches en terminologie ont marqué un grand intérêt pour les marques de figement qui ne concernent pas des syntagmes nominaux et ci-après désignées par le générique expression idiomatique ${ }^{1}$. L'émergence du concept de langue de spécialité (L.Sp.) confirme cette évolution qui tend à mieux situer le terme spécialisé dans une forme d'énoncé qui lui serait propre. Divers colloques et séminaires ont été ou seront prochainement consacrés à l'étude des phénomènes syntagmatiques. On citera notamment le colloque organisé par l'École de traduction et d'interprétation de l'Université de Genève (Bessé 1992).

Force nous est pourtant de constater avec Heid et Freibott (1991: 84-85) que les concepteurs de logiciels de gestion de données terminologiques n'ont guère tenu compte des préoccupations actuelles: le traitement de l'idiomaticité fait figure de parent pauvre si l'on en juge d'après les produits présents sur le marché. Notre propos vise plus particulièrement à étudier les problèmes de description des marques de figement dans un logiciel qui adopte une perspective notionnelle. Il rend compte d'une recherche menée dans le cadre du développement du logiciel Termisti, dont les fondements théoriques et les principes de consultation ont déjà été décrits ailleurs ( $c f$. bibliographie).

Le lieu et l'espace impartis ne nous permettent pas d'entrer dans des discussions portant sur le statut d'unité terminologique des expressions idiomatiques ${ }^{2}$. Notre démarche se fonde sur une approche pragmatique de leur traitement, dans le contexte descriptif des bases de données relationnelles. Loin de vouloir totalement évacuer le problème du statut, nous pensons qu'il y a lieu de traiter les expressions comme des unités terminologiques dès lors qu'elles véhiculent un contenu notionnel propre et qu'elles exigent des traductions particulières. Cette conviction, partagée par certains orateurs du colloque de Genève ${ }^{3}$, se fonde essentiellement sur divers problèmes d'équivalence rencontrés en terminologie mutilingue et ici exposés.

\section{PROBLÉMATIQUE}

Traditionnellement, les bases de données contiennent des fiches fournisșant une information à propos de termes désignant des notions particulières qui, le plus souvent, 
concernent des réalités concrètes ou abstraites. Plus rarement, le terme est un verbe, un adjectif ou un adverbe désignant une action particulière, un état, une manière, etc. On lie souvent cette diversification syntaxique à des langues de spécialités marquées par l'histoire et/ou liées à de vastes domaines de connaissance ${ }^{4}$. Ainsi en va-t-il, par exemple, du domaine de la navigation retenu pour cet exposé.

Dans la réalité de son travail, le terminologue est parfois confronté à la nécessité de décrire des termes simples qui ne relèvent pas de la catégorie nominale. Dès lors que ces termes simples véhiculent un contenu notionnel propre, il n'hésite guère à leur consacrer des fiches particulières et veille à fournir des contextes d'usage. Paradoxalement, lorsqu'il est confronté à une suite lexicale récurrente, qui ne répond pas aux caractéristiques du syntagme nominal, il hésite à décrire comme terme spécialisé telle locution verbale, adjective ou adverbiale.

Cette attitude prudente est liée au fait que les expressions idiomatiques, quelle que soit leur nature, utilisent généralement des termes désignant des notions précises, déjà décrites ailleurs dans la base de données. Certes, tant que la description ne concerne qu'une seule langue, on peut être tenté de n'utiliser que les rubriques «contexte» et «note», voire de créer une rubrique "phraséologie», pour décrire ce que l'on tend à considérer comme des usages particuliers d'un terme déjà mentionné. Il en va de même lorsque, par chance, les expressions de la langue source et de la langue cible utilisent des termes parfaitement équivalents. Toutefois, dès lors que l'on prend plus de deux langues en compte, cette parfaite correspondance devient exceptionnelle, ce qui pose de gros problèmes de traduction dans une base de données de type relationnel.

Les gestionnaires terminologiques qui, comme MC4 ou Termisti, suivent une perspective notionnelle multilingue, appartiennent à ce type de base. Chaque notion y correspond à un numéro servant de lien entre des fiches qui décrivent les termes de chaque langue; les équivalences sont fournies grâce à la mise en relation des fiches qui possèdent le même numéro, de telle sorte que tous les couples de langues soient envisageables ${ }^{5}$. Or, dans la pratique, on sait qu'une expression se traduit rarement de manière littérale, ce qui implique que l'expression équivalente en langue cible ne pourra pas figurer sous chacun des équivalents des termes utilisés par la langue source. Ainsi, en navigation, la traduction anglaise de à sec de toile (= under bare poles) ne saurait, en bonne doctrine notionnelle, figurer sous les termes $\grave{a} \sec (=d r y)$ ou toile (= canvas).

\section{PRINCIPAUX PROBLÈMES D'ÉQUIVALENCE}

Pour décider du traitement des expressions idiomatiques à appliquer dans Termisti, il nous a fallu rassembler des cas de figure et réfléchir à la meilleure manière de les intégrer dans une approche notionnelle. L'inventaire proposé ci-après est plus particulièrement fondé sur l'étude d'un corpus de termes relevant du domaine de la marine marchande ${ }^{6}$ et ne pourrait prétendre épuiser tous les phénomènes idiomatiques. Il a conduit à une option de traitement terminologique susceptible d'être ultérieurement amendée.

\section{Expressions équivalentes ne recourant pas aux mêmes notions}

Il est fréquent que, d'une langue à l'autre, l'expression idiomatique ne fasse pas appel à des termes désignant la même notion: les équivalences de l'ordre de manœuvre anglais mainsail haul! constituent une très bonne illustration de ce phénomène, puisque ni le français ni l'allemand n'usent d'une traduction littérale. La seule prise en compte de l'anglais et du français implique au moins cinq notions différentes.

[1] mainsail haul!

[2] to brace about changez derrière!

changer rund achter!

herumbrassen 

[3] to shift
[4] to haul
[5] astern
[6] mainsail

\author{
changer \\ haler \\ de l'arrière, derrière \\ grand-voile
}

wechseln

holen

hinteraus, über Steuer, achteraus

Grossegel

Quelle que soit la langue envisagée, il serait aberrant de faire figurer cette injonction comme un simple contexte des termes (notions) qu'elle utilise. Une telle attitude équivaudrait, en effet, à interdire toute découverte d'une équivalence au sein de la base de données, puisque, par exemple, l'équivalent grand-voile de mainsail ne concerne en rien la traduction de mainsail haul! (tableau ${ }^{\circ} 1$ ).

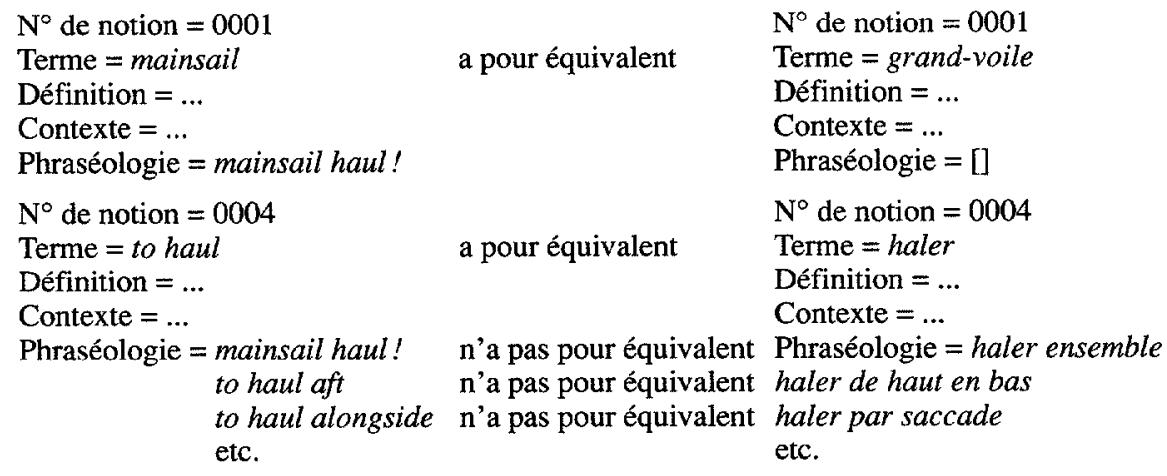

Tableau $\mathbf{n}^{\circ} 1$

Notre option est de considérer que de nombreuses expressions constituent de véritables unités de sens que nous nommons notions idiomatiques et que nous décrivons comme telles dans des fiches particulières. En l'occurrence, les expressions mainsail haul!, changez derrière! et rund achter! sont traitées à la manière de n'importe quelle autre unité terminologique. Elles reçoivent donc chacune une entrée particulière, renvoyant à un même numéro de notion qui établit leur équivalence.

Un tel principe n'est guère coutumier dans les dictionnaires, de sorte que le traducteur risque de ne pas découvrir la fiche adéquate, le mouvement habituel de consultation consistant à rechercher une expression à partir d'un terme jugé important en son sein 7 . Deux solutions permettent d'éviter ce danger. La première consiste à rechercher tous les syntagmes où figure un même mot. Ce principe de recherche a été prévu dans le logiciel Termisti, de sorte que l'on peut isoler tous les termes composés relevant d'une même famille lexicale. Inévitablement, cette fonction de recherche engendre beaucoup de bruit lorsqu'on l'utilise pour identifier une expression idiomatique. Par exemple, le dictionnaire de Paasch (1901) ne comporte pas moins de dix entrées utilisant le verbe to haul et pas moins de cinq utilisant le mot mainsail.

La deuxième solution a notre préférence et se rapproche de celle proposée par Heid et Freibott (1991: 87). Elle consiste à prévoir une fonction de recherche qui établirait une correspondance entre une expression idiomatique et les termes qu'elle utilise. Ce lien idiomatique n'a de valeur qu'au sein d'une seule et même langue et n'implique jamais un synonyme. En cela, il est très différent des liens notionnels, qui, eux, réunissent des notions et sont donc valables pour tous les synonymes, dans toutes les langues envisagées. Il possède à la fois une nature lexicale, car il renvoie à un terme qu'il contient, et une nature sémantique, car, pour effectuer ce renvoi, il faut distinguer les éventuels homonymes. Ainsi, le verbe changer désigne deux notions différentes (exemples nos 2 et 3 ), dont seule la 
première concerne l'expression changez derrière! Le tableau $n^{\circ} 2$ illustre l'établissement de ce lien idiomatique.

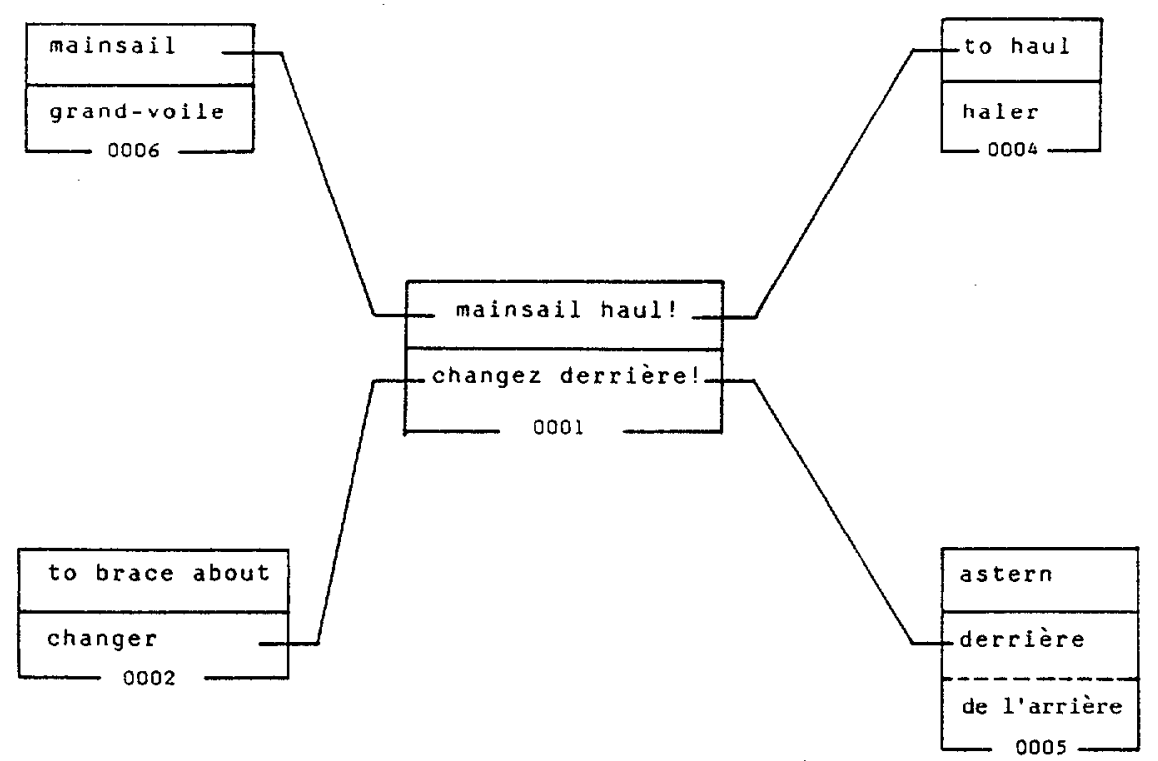

Tableau $\mathbf{n}^{\circ} 2$

L'équivalent français changez derrière! de la notion idiomatique 1 sera lié aux termes français changer (notion 2) et derrière (notion 5), tandis que l'équivalent anglais mainsail haul! sera, lui, lié aux équivalents anglais mainsail (notion 6) et to haul (notion 4). Bien entendu, les synonymes éventuels (de l'arrière) ne sont pas concernés par le lien.

\section{Expressions équivalentes recourant à une même notion}

Parfois, les expressions idiomatiques équivalentes font référence à au moins une même notion dans chacune des langues envisagées. Ainsi, dans les exemples suivants :

$$
\begin{aligned}
& \text { [7] to sound the pumps } \\
& \text { [8] to row a long stroke } \\
& \text { [9] to set sail } \\
& \text { [10] slack in stays }
\end{aligned}
$$

\author{
die Pumpen peilen \\ Mit langem Schlag rudern \\ Segel setzen \\ langsam beim Wenden
}

Face à de telles expressions, la qualité du travail de dépouillement terminologique se trouve fortement engagée. En effet, il faut découvrir que les collocations récurrentes sonder la cale, allonger la nage, mettre à la voile ou lent à virer constituent des expressions idiomatiques qui méritent un traitement notionnel, dans la mesure où elles possèdent chacune une acception propre et appellent des traductions précises. Dans un cas comme celui de sonder la cale (exemple $n^{\circ} 7$ ), rien n'interdirait de se contenter de la fiche faisant référence à la notion commune (sonder), pour autant que le traducteur consulte celle-ci. Cette procédure demeure valable tant que n'intervient pas une nouvelle langue qui ferait référence à une autre notion (slack in stays ${ }^{8}$ ) ou que la traduction littérale ne s'avère pas impossible (allonger la nage, mettre à la voile). Ces dangers potentiels nous portent à préférer la solution de la notion idiomatique, qui, seule, garantit la mention de la juste traduction en français dans les exemples nos 8, 9 et 10 . 


\section{Équivalence entre une expression idiomatique et un terme simple}

Il arrive qu'une notion désignée par un terme simple dans une langue ait pour équivalent une expression idiomatique dans une autre langue, comme cela se produit dans les exemples suivants.

$\begin{array}{lll}\text { [11] to make leeway } & \text { dériver } & \text { abtreiben } \\ \text { [12] to run ashore } & \text { s'échouer } & \text { auf Strand laufen } \\ \text { [13] to founder head down } & \text { sancir } & \text { Kopf über sinken } \\ \text { [14] to broach } & \text { partir au lof ; faire chapelle } & \text { eine Eule fangen }\end{array}$

La solution du lien idiomatique semble particulièrement indiquée pour rendre compte d'une telle situation. Les termes simples attestent que l'on a bel et bien affaire à des notions - représentant ici des actions - dont les équivalents sont des expressions comme to make leeway ou partir au lof. Elles pourront être reliées par le lien idiomatique avec les termes concernés: leeway, ashore, lof. Ces cas justifient pleinement l'appellation notion idiomatique retenue ci-dessus: si des verbes comme dériver ou to broach peuvent faire l'objet d'une description notionnelle, il doit en être de même de leurs équivalents, fussent-ils conditionnés par la phraséologie.

\section{Plusieurs expressions d'une même langue recourent à une même notion}

Notre attention a été particulièrement retenue par un phénomène fort intéressant: il arrive que dans certaines langues, diverses expressions idiomatiques fassent toujours référence à une même notion, alors que cette constante n'apparaît pas dans d'autres langues.
[15] to lay the keel of a vessel
[16] to lay a deck
[17] to lay alongside the quay
[18] to lay a rope
mettre la quille d'un navire border un pont placer le long du quai commettre un cordage
[19] to lay on a metal sheating doubler un navire en métal ein Deck legen
den Kiel eines Schiffes legen längsseit der Kaje legen ein Tau schlagen eine Metallhaut anlegen

Un pareil cas pose le problème du dégroupement homonymique de la notion récurrente. En effet, la logique du traitement retenu veut que l'on crée une notion idiomatique pour chaque expression et que l'on utilise le lien idiomatique déjà évoqué pour opérer des rattachements avec la notion commune (ici, to lay). En réalité, celle-ci appartient le plus souvent à la langue générale et son sens comme sa traduction varient avec le contexte. Il paraît quelque peu vain de créer une telle notion dont les équivalents seraient particulièrement nombreux. En outre, ils renverraient à des réalités (des notions) très différentes, en sorte qu'il est impossible de proposer une définition unique, répondant à l'exigence de monosémie (exemple $\mathrm{n}^{\circ} 20$ ).

$$
\text { [20] to lay legen, anlegen, schlagen }
$$

L'application du principe de dégroupement homonymique propre aux bases de données notionnelles multilingues ${ }^{9}$ - pour rendre compte de toutes les équivalences possibles - engendrerait une nette multiplication des entrées.

$\begin{array}{lll}{[21] \text { to lay }} & \text { mettre } & \text { legen } \\ {[22] \text { to lay }} & \text { border } & \text { legen } \\ {[23] \text { to lay }} & \text { placer } & \text { legen } \\ {[24] \text { to lay }} & \text { commettre } & \text { schlagen } \\ {[25] \text { to lay }} & \text { doubler } & \text { anlegen }\end{array}$

Cette dernière conséquence est en contradiction avec l'octroi d'une entrée propre aux notions idiomatiques. En effet, les notions proposées ci-dessus ne se distinguent que dans 
le cadre contextuel de l'idiomaticité; en l'occurrence, par une information valencielle à propos de leur complément. Le plus simple semble donc de faire figurer cette information en vedette. Ce choix est d'autant plus pertinent que, dans le domaine de la navigation, le verbe to lay n'a pas d'existence autonome dès lors qu'on l'envisage sous l'angle de la traduction et ne mérite guère une entrée propre. Selon le contexte, il renvoie à des notions différentes et régit des traductions particulières.

Le choix de la notion idiomatique est donc renforcé par l'analyse de cette situation, à vrai dire fréquente. Il en va, par exemple, de même pour l'usage de nombreuses prépositions:
[26] by land
[27] by sea
[28] by the bulk
[29] by the day
[30] by the head
[31] by the log
[32] by the piece
[33] by the stern
[34] by the wind

par terre
par mer
en gros
à la journée
sur nez
à l'estime
à la pièce
sur cul, sur l'arrière
à la bouline

zu Lande
zu Wasser
im Ganzen
pro Tag
Vorlastig
nach Gissung
pro Stück
achterlastig, hinterlastig
beim Winde

Réserver à by une entrée particulière supposerait la création d'un grand nombre de notions homonymes, alors qu'il est bien plus simple de situer chaque traduction dans son contexte et de définir chaque fois le concept véhiculé. En l'occurrence, il importe plus d'expliquer ce que recouvre la notion by the log et de fournir son équivalent à l'estime que de préciser que by se traduit par $a$ dans certaines expressions ${ }^{10}$. Lors de l'élaboration de la base de données, le terminologue doit, en effet, réfléchir à l'attitude qui lui paraît la plus efficace pour rendre compte de l'usage et répondre aux besoins du traducteur profane.

\section{Expression idiomatique utilisant une notion sans équivalent}

Nous avons rencontré des cas où l'expression idiomatique d'une langue donnée englobe systématiquement une notion qui n'existe pas comme telle dans les autres langues.

$\begin{array}{lll}{[35] \text { bound }} & {[]^{11}} & {[]} \\ {[36] \text { weather bound }} & \text { arrêté par le gros temps } & \text { durch Unwetter zurückgehalten } \\ {[37] \text { wind bound }} & \text { arrêté par le vent } & \text { durch den Wind aufgehalten } \\ {[38] \text { ice bound }} & \text { retenu par les glaces } & \text { im Eise besetzt }\end{array}$

Ainsi, le mot anglais bound, commun à de nombreuses expressions propres au déplacement sur mer, ne correspond à aucune notion équivalente en français et en allemand. Il s'agit d'un morphème lié qui n'apparaît que dans des expressions idiomatiques particulières. Quand bien même on choisirait de décrire bound comme une notion propre à la langue anglaise, il n'y aurait pas lieu de rattacher cette notion aux expressions idiomatiques françaises et allemandes. Ce constat conforte notre choix de limiter l'usage du lien d'idiomaticité à une seule et même langue.

\section{Les expressions ne contiennent aucun terme du domaine}

Sans préjuger de l'existence d'autres phénomènes, nous citerons pour dernière problématique le cas où les expressions ne contiennent aucun terme propre à la spécialité, mais véhiculent un contenu particulier au sein du domaine envisagé.

[39] to turn turtle

[40] banking the fires faire chapeau

pousser les feux au fond des grilles
[] aufbänken der Feuer 
De telles expressions ne peuvent être rangées sous aucune autre entrée du domaine. Même dans un système qui aurait pour principe de ne pas consacrer une fiche particulière aux expressions idiomatiques, elles devront nécessairement recevoir une entrée autonome.

\section{Une réalité complexe}

Bien entendu, les quelques phénomènes ici décrits peuvent s'entremêler. Ainsi, dans les exemples nos 41 et 42 , les expressions anglaises et allemandes relèvent du cas décrit dans le paragraphe précédant alors que les équivalents français sont des termes simples comme dans le paragraphe sur l'équivalence entre une expression idiomatique et un terme simple.

$\begin{array}{lll}\text { [41] to approach the land } & \text { atterir } & \text { sich dem Lande nähern } \\ \text { [42] to go clear } & \text { parer } & \text { klar kommen }\end{array}$

Une telle observation justifie encore davantage le choix de décrire comme des notions des phénomènes qui, a priori, relèvent de la seule description phraséologique. En effet, le principe de la notion idiomatique devrait garantir l'homogénéité macrostructurelle du corpus, la plupart des expressions étant traitées de la même manière.

\section{RÉALISATION INFORMATIQUE}

Une première implantation du lien idiomatique dans le logiciel Termisti a été réalisée par notre collègue Philippe Petrussa. L'encodage de la relation se réalise par un processus voisin de celui utilisé pour les liens notionnels (on retrouve notamment l'usage du système de double prédicat). Le terminologue déclare vouloir établir un lien d'idiomaticité et sélectionne une expression qu'il a préalablement décrite dans une fiche. Le programme réalise alors un parsage élémentaire pour identifier les constituants de l'expression qui correspondent à des termes déjà présents dans le micro-glossaire concerné ${ }^{12}$. Il suffit ensuite au terminologue de valider les liens jugés pertinents ou, en l'absence de réponse valable, d'entrer lui-même le terme adéquat (tableau $\mathrm{n}^{\circ} 3$ ).

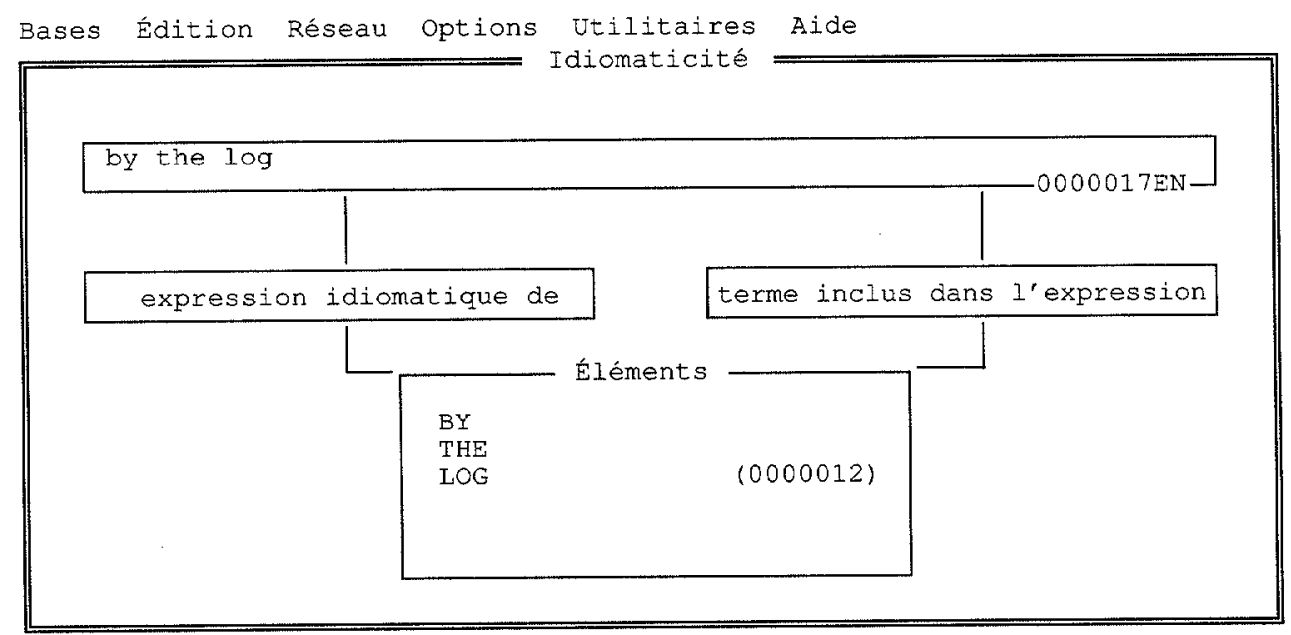

1 Termisti - EN->Er

Tableau $n^{\circ} 3$ 
La consultation se réalise à partir de la liste des termes présents dans la base de données. Grâce à un raccourci clavier, on peut indifféremment rechercher la ou les expression(s) idiomatique(s) qui utilisent tel terme ou appeler le ou les terme(s) utilisé(s) par telle expression idiomatique. Dès que la réponse est obtenue, il est bien sûr possible de consulter la fiche se rapportant au terme ou à l'expression concernée et dont la traduction apparaît au bas de l'écran (tableau $\mathrm{n}^{\circ} 4$ ).

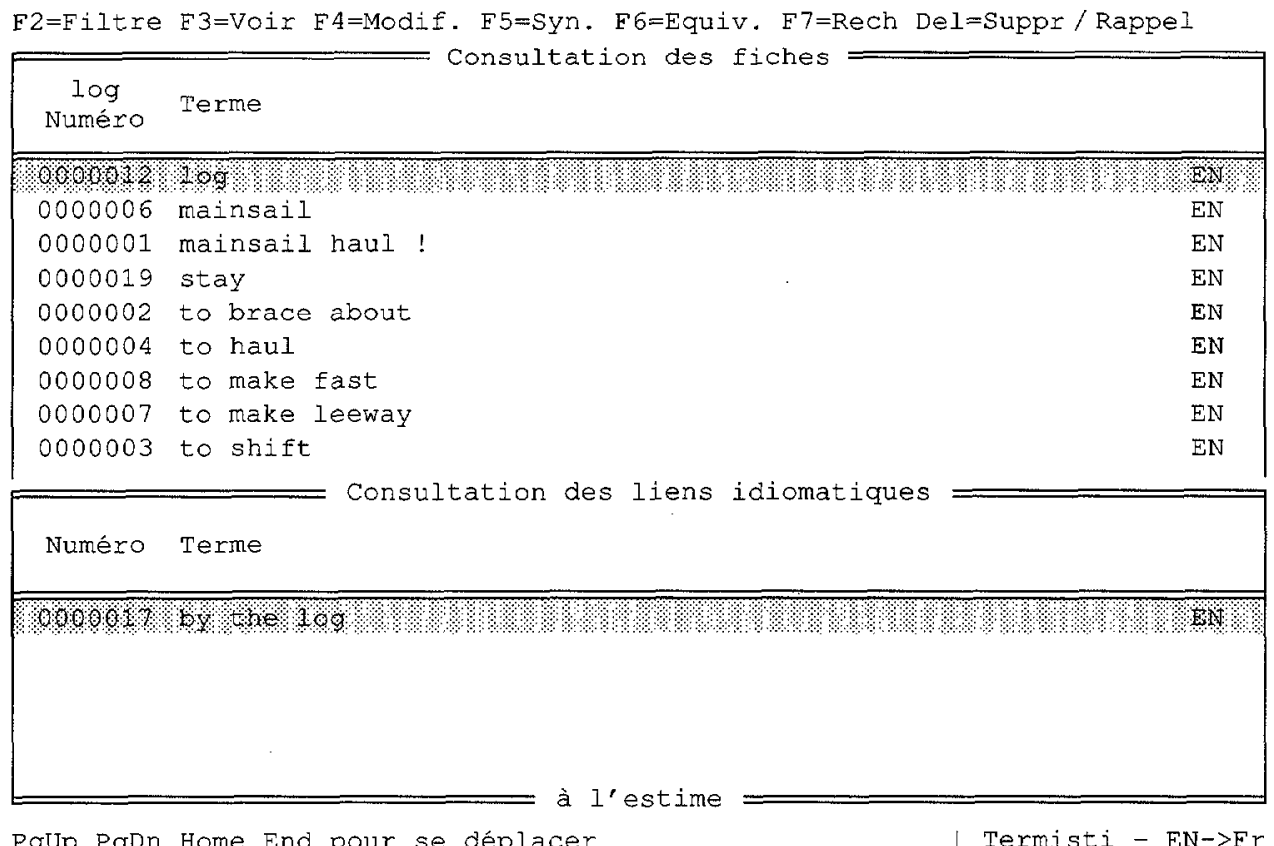

\section{Tableau $n^{\circ} 4$}

\section{USAGES DU LIEN IDIOMATIQUE}

La première fonction du lien idiomatique est, bien sûr, de permettre au traducteur de découvrir les usages d'un terme technique qui sont fortement conditionnés par la phraséologie. Partant d'un terme à traduire, il peut vérifier si le contexte observé en langue source correspond à une expression idiomatique réclamant une traduction particulière.

La démarche inverse, qui consiste à découvrir un terme à partir d'une expression idiomatique, ne paraît pas propre à l'acte de traduction: elle relève d'une approche plus cognitive visant à mieux connaître une langue de spécialité. Comme on l'a déjà affirmé, l'idiomaticité naît souvent de l'usage; elle s'explique alors par l'histoire du domaine scientifique ou technique et par l'évolution de sa langue. En ce sens, une expression idiomatique peut inclure un terme désignant une notion qui, en synchronie, n'a plus grand rapport avec elle. Ainsi, l'expression à la bouline (exemple $\mathrm{n}^{\circ} 34$ ) signifie «le plus près possible de l'axe du vent». Progressant de cette expression vers le terme bouline, le traducteur profane découvre sa motivation ancienne : la bouline est un cordage qui sert à ouvrir la chute au vent (le côté d'où vient le vent) d'une voile carrée pour permettre au navire de mieux se rapprocher de l'axe du vent et donc de mieux louvoyer. 


\section{CONCLUSION}

La construction phraséologique offre quelquefois des régularités qui permettent de décrire une expression idiomatique en ne recourant qu'à la mention de contextes bien choisis, agrémentés d'une note éventuelle. Toutefois, le traitement ici proposé se fonde sur le caractère exceptionnel d'équivalences aussi régulières. Il tient compte des difficultés de traduction qu'implique la prise en compte de l'idiomaticité dans une base de données comportant un grand nombre de langues. De ce point de vue, la création d'une fiche particulière traitant de l'expression idiomatique comme s'il s'agissait d'une véritable notion permet une gestion rigoureuse et homogène, apte à rendre compte de la diversité des problèmes d'équivalences.

Quand bien même elle est dotée d'un sémantisme propre, la notion idiomatique ne semble guère intégrée dans le tissu des relations notionnelles qui caractérise nombre de sous-domaines. Si la mise en place du lien idiomatique suppose une analyse sémantique en cas d'homonymie de l'un des constituants, il reste que son caractère lexical le distingue franchement du lien notionnel qui, dans notre conception, est indépendant des langues (Merten et al. 1993: 284-289). De par cette nature distincte, le lien idiomatique relève moins de la terminologie que de la lexicologie. Son utilité patente atteste de la place qui doit être accordée à cette dernière dimension au sein d'un système de gestion terminologique destiné au traducteur.

\section{Notes}

1. Les auteurs parlent de phraséologie, de collocation, de marque de figement ou encore d'idiomaticité, toutes appellations que nous n'entendons point discuter ici.

2. Heid et Freibott (1992) ont dressé un très intéressant état de la question.

3. Nous pensons notamment aux points de vue exprimés par Gerhard Budin et Christian Galinski (1992) ainsi que par Ulrich Heid (1992).

4. On notera que les bases de données élaborées à l'aide du logiciel Termisti dans le cadre du projet de recherche Élaboration de micro-glossaires informatisés pour les langues de spécialité ne comportent quasi aucune expression idiomatique. Les domaines abordés concernent la phytovirologie, la pédologie, la télédétection aérospatiale et les effets de serre.

5. Sur la structure générale des bases de données relationnelles, lire Le Meur (1993: 255-258); la structure de Termisti est décrite par Merten et al. (1993). On notera, par ailleurs, que certains logiciels ne permettent que d'utiliser un nombre très restreint de langues, avec - en outre - une limitation des combinaisons de langues.

6. Les exemples cités ci-après appartiennent au domaine de la navigation à voile telle que pratiquée au début du siècle. Ils sont, pour la plupart, extraits du dictionnaire de Paasch (1901) que nous étudions dans le cadre d'une thèse de doctorat en science du langage (Université Paris-Nord). Exploité tel quel, ce corpus contient des formes surannées, voire des équivalences discutables.

7. Béjoint et Thoiron (1992: 522) soulignent d'ailleurs le poids de l'habitude : «Jeter les pratiques lexicographiques traditionnelles pour leur substituer des méthodes fondées sur des classifications inusitées jusque-là risque de prendre au dépourvu les utilisateurs.»

8. Littéralement, slack in stays = «mou dans les étais»: lorsque les étais ne sont pas tendus, le navire se rapproche difficilement de l'axe du vent.

9. À propos du principe de dégroupement homonymique dans une base de données notionnelles multilingue, lire Van Campenhoudt (1991).

10. Notre point de vue diverge ici de celui de Heid et Freibott (1992: 88-89) qui proposent de conserver la notion polysémique et suggèrent que ce soit la consultation du lien d'idiomaticité qui permette de choisir l'équivalent adéquat parmi toutes les traductions proposées.

11. Signifie que le dictionnaire de référence ne fournit pas d'équivalence.

12. Dans l'état actuel du logiciel, cette correspondance s'établit d'après une simple reconnaissance formelle. 


\section{RÉFÉRENCES}

BÉJOINT, Henri et Philippe THOIRON (1992) : «Macrostructure et microstructure dans un dictionnaire de collocations en langue de spécialité», dans Bessé (1992: 513-522).

BESSÉ, Bruno de (Éd.) (1992) : Phraséologie et terminologie en traduction et en interprétation, Actes du colloque international de Genève, 2-4 octobre 1991, Terminologie et traduction, 2 / 3.

BLAMPAIN, Daniel (1992) : «Traduction et écosystèmes terminologiques», dans Bessé (1992: 457-466).

BLAMPAIN, Daniel, PETRUSSA, Philippe et Marc VAN CAMPENHOUDT (1992): «À la recherche d'écosystèmes terminologiques», dans Clas et Safar (1992: 273-282).

BUDIN, Gerhard et Christian GALINSKI (1992): «Übersetzungsorientierte Phraseologieverwaltung in Terminologiedatenbanken», dans Bessé (1992: 565-574).

CLAS, André et Hayssam SAFAR (Éds.) (1992): L'environnement traductionnel. La station de travail du traducteur de l'an 2001, Actes des Deuxièmes Journées scientifiques du Réseau thématique de recherche «Lexicologie, terminologie et traduction», Mons, 25-27 avril 1991, Sillery et Montréal, Presses de 1'Université du Québec et AUPELF-UREF.

Centre nautique des Glenans (1990) : Cafouiller en anglais = Messing about in French, Paris et Dublin, Centre nautique des Glénans (inédit).

GOUADEC, Daniel (Éd.) (1993) : «Terminologie \& terminotique : outils, modèles \& méthodes», Actes de la première université d'automne en terminologie, Rennes 2, 21-26 septembre 1992, Paris, La Maison du dictionnaire.

HEID, Ulrich (1992) : «Décrire les collocations : deux approches lexicographiques et leur application dans un outil informatisé», dans Bessé (1992: 523-548).

HEID, Ulrich et Gerhard FREIBOTT (1991) : «Collocations dans une base de données terminologique et lexicale», Meta, 36-1, pp. 77-91.

LE MEUR, André (1993): «Modèles, structures et échanges de données terminologiques», dans Gouadec (1993: 245-274).

L'HOMME, Marie-Claude (1992): «Les unités phraséologiques verbales et leur représentation en terminographie», dans Bessé (1992: 493-503).

MERTEN, Pascaline, MERTENS, Jean et Marc VAN CAMPENHOUDT (1993): «Microglossaire, réseau notionnel et gestion informatique. Une expérience de recherche en Communauté française de Belgique», dans Gouadec (1993: 277-293).

PAASCH, Heinrich (1901): From Keel to Truck = De la quille à la pomme du mât = Vom Kiel zum Flaggenknopf, $3^{\mathrm{e}}$ édition, Anvers, chez l'auteur et Hambourg, Eckardt \& Messtorff.

PAVEL, Silvia: Bibliographie de la phraséologie (1905-1992), Montréal, Secrétariat d'État du Canada (Direction de la terminologie et des services linguistiques) et Rint (inédit).

VAN CAMPENHOUDT, Marc (1991): «TI, le logiciel d'expérimentation notionnelle de Termisti», Terminologies nouvelles, 5, pp. 11-14. 